\title{
Review
}

\section{Recent Progress in Understanding the Mode of Action of Acetylxylan Esterases}

(Received February 26, 2014; Accepted March 14, 2014)

(J-STAGE Advance Published Date: March 25, 2014)

\author{
Peter Biely, ${ }^{1, *}$ Bjørge Westereng, ${ }^{2}$ Vladimír Puchart, ${ }^{1}$ Pieter de Maayer ${ }^{3}$ and Don A. Cowan ${ }^{3}$ \\ ${ }^{1}$ Institute of Chemistry, Center of Glycomics, Slovak Academy of Sciences \\ (Dúbravská cesta 9, 84538 Bratislava, Slovakia) \\ ${ }^{2}$ Department of Chemistry, Biotechnology and Food Science, Norwegian University of Life Sciences \\ (P.O. Box 5003, NO-1432, Aas, Norway) \\ ${ }^{3}$ Centre for Microbial Ecology and Genomics, University of Pretoria (0028 Hatfield, Pretoria, South Africa)
}

\begin{abstract}
Acetylation is one of the main obstacles to the effective enzymatic conversion of hemicelluloses to fermentable sugars. In nature, the microbial degradation of hemicellulose involves the action of deacetylating esterases that act synergistically with glycoside hydrolases. In the industrial processing of lignocelluloses biomass, alkaline pretreatments remove acetyl groups by saponification, but other non-alkaline pretreatment methods generate acetylated hemicelluloses. Complete saccharification of plant hemicelluloses can't be achieved without the deacetylating enzymes. Recent years have witnessed considerale progress in our understanding of the mode of acetylation of hemicellulose and mode of action of microbial polysaccharide deacetylases. In this article we focus on the diversity and role of acetylxylan esterases in the breakdown of acetylxylan, the most abundant hemicellulose in nature.
\end{abstract}

Key words: plant biomass conversion, xylan, acetylation, acetylxylan esterases, mode of action

\section{INTRODUCTION}

The major obstacle of efficient and economically feasible bioconversion of renewable plant biomass is its recalcitrance as a result of complexity of the plant cell wall structure. The structural complexity is, in part, the result of plant evolution under strong pressure to enhance the resistance to microbial invaders. With the exception of insoluble cellulose, almost all other cell wall polysaccharide components, mainly hemicelluloses and pectin, are to some degree branched and partially esterified with acetic acid. ${ }^{1,2)}$ Esterification of the side chain sugar residues of polysaccharides with acetyl groups is apparently critical in determining the physical properties of the cell wall, although other polymer modifications are also important; for example, esterification with phenolic acids plays a role in cross-linking., ${ }^{3,4}$ Here we review recent progress in understanding of enzymatic degradation of native acetylated hemicellulose using NMR and MS approaches. Particular attention is paid to the breakdown of partially acetylated hardwood glucuronoxylan, as studies of the action of deacetylating carbohydrate

* Corresponding author (Tel. +4212-5941-0275, Fax. +4212-5941-0222, E-mail: chempbsa@savba.sk).

Abbreviations: AcXE, acetylxylan esterase; GH, glycoside hydrolase; $\mathrm{CE}$, carbohydrate esterase; DMSO, dimethyl sulfoxide; Xylp, D-xylopyranose or D-xylopyranosyl; MeGlcA, 4-O-methyl-D-glucuronic acid; MeGlcA ${ }^{3} \mathrm{Xyl}$, , 4-O-methyl-D-glucuronosyl- $\alpha$-1,2-D-xylopyranosyl$\beta$-1,4-D-xylopyranosyl- $\beta-1,4-\mathrm{D}$-xylopyranose (the upper index indicates the number of the xylosyl residue from the reducing end substituted with MeGlcA); XylxAcy, acetylated $\beta$-1,4-xylooligosaccharide containing $\mathrm{x}$ xylose residues and y acetyl groups; MeGlcAXylxAcy, acetylated aldouronic acid containing one MeGlcA, x xylose residues and y acetyl groups; MeGlcA2XylxAcy, acetylated aldouronic acids containing two MeGlcA residues. esterases have principally focused on this polysaccharide.

\section{ROLE OF ACETYLATION OF PLANT CELL WALL POLYSACCHARIDES}

Acetylation has been clearly shown to hinder the action of microbial glycanases. ${ }^{5}$ However, acetylation also dramatically changes the physico-chemical properties of polysaccharides. While full acetylation makes polysaccharides water-insoluble, partial acetylation, as observed in plant xylans, increases their solubility in water. Partial acetylation increases the bulk volume of the polysaccharide due to a higher degree of hydration. This is likely to have important consequences for transport of water and nutrients through the plant cell walls and also increase their susceptibility to enzymatic hydrolysis. This idea finds support in recent reports that the knock-out of acetyl transferase genes in Arabidopsis resulted in a lower degree of hemicellulose acetylation, enhanced the recalcitrance of the cell wall and the plant resistance to infection by the necrotrophic fungal pathogen Botrytis cinerea. ${ }^{6-8)}$ Acetyl groups in the main xylan chain obviously prevent closer association of xylan with cellulose, thus increasing the plant resistance to pathogen attack.

It is not known at which stage of plant evolution the acetylation of polysaccharides was introduced, however, it is likely that deacetylating enzymes evolved in parallel with microorganisms invading plants or proliferating on plant residues. The substrate specificity of some carbohydrate esterases for elements of the polysaccharide main chain suggests that some of these enzymes evolved from glycoside hydrolase ancestors. ${ }^{5,9-11)}$ 


\section{PRETREATMENTS AFFORDING ACETYLATED HEMICELLULOSES}

The bioconversion of insoluble plant biomass to soluble fermentable sugars is an essential step in the production of liquid fuels and a variety of other chemicals and useful products including food additives. Typically, the first and crucial step of this bioconversion process is the mechanical and physico-chemical pretreatment to render the lignocellulosic biomass susceptible to enzymatic saccharification. Alkaline pretreatment of biomass results in saponification of ester linkages, therefore, here we will consider only the acetylated hemicellulose fractions obtained under nonalkaline conditions, such as steam explosion, hot water treatment and autohydrolysis at increased pressure and temperature. ${ }^{12-17)}$ Hemicellulose also remains acetylated after ionic liquid, organosolvent or super-critical $\mathrm{CO}_{2}$ pretreatments. ${ }^{15-17)}$

\section{PATTERNS OF XYLAN ACETYLATION IN PLANTS}

Acetylation is the most common esterification of plant xylans. In hardwood glucuronoxylans more than half of the main chain xylopyranosyl (Xylp) residues are acetylated. ${ }^{18-19)}$ The majority of Xyl $p$ residues are singly acetylated at positions 2 or 3 , but a smaller fraction is also doubly 2,3-acetylated. There are also reports of 3-O-acetylation of $\mathrm{Xyl} p$ residues substituted with 4- $O$-methyl-D-glucuronic acid residues (MeGlcA). ${ }^{18-21)}$ Similar acetylation patterns have been observed for Xyl $p$ residues substituted by GlcA. ${ }^{8)}$ Several lines of evidence suggest that there could be several types of glucuronoxylan, which differ in their content of MeGlcA and acetyl groups. ${ }^{8,22}$ We have shown that a DMSO-extracted beechwood acetylglucuronoxylan contains a much lower content of MeGlcA residues than alkali extracted polymer (M. Vršanská and P. Biely, unpublished results), suggesting that the reported values of the degree of substitution with MeGlcA and the degree of acetylation are average values. Acetylation of $\mathrm{Xyl} p$ residues has been confirmed in cereal arabinoglucuronoxylan ${ }^{20)}$ and in xylan from Arabidopsis thaliana. ${ }^{23)}$ Although only monoacetylation of Xyl $p$ residues was reported in cornstalk arabinoxylan, ${ }^{20)}$ it has clearly been demonstrated that the acetylation features of the hardwood polysaccharide are also seen in dimethylsulfoxide extracted acetylglucuronoxylan from Arabidopsis. ${ }^{24)}$ As in the hardwood, Xylp residues in Arabidopsis xylan are mono- and diacetylated at positions 2 and 3, and also 3-O-acetylated on Xyl $p$ residues 2- $O$-substituted with uronic acid. ${ }^{24)}$ The distinct feature of the Arabidopsis xylan is that in addition to MeGlcA it also contains GlcA side residues. ${ }^{8)}$ A detailed structural analysis of one of the most recalcitrant plant xylans occurring in corn fiber ${ }^{25}$ ) revealed novel sites of polysaccharide acetylation. ${ }^{26)}$ Acetyl groups were found at position 2 in approximately half of $\mathrm{Xyl} p$ residues. Some of these Xyl $p$ residues carry at position 3 Ara $f$ residues esterified at position 5 with ferulic acid or substituted at position 2 with additional Xyl $p$ residue or with the disaccharide 2-O- $\alpha$-D-galactopyranosyl-Xyl $p{ }^{26)}$ The known patterns of acetylation of hardwood and cereal xylans, and the xylan of annual plants, are depicted in Fig. 1.

\section{EFFECT OF XYLAN ACETYLATION ON XYLAN HYDROLYSIS BY ENDOXYLANASES}

The inhibition of endoxylanase-catalyzed hydrolysis of xylan by its acetylation is illustrated in Table 1. As shown by a MALDI ToF MS analysis of oligosaccharides released from acetylated xylan and its deacetylated counterpart, the number of products generated from acetylated polysaccharides by family 10 or family 11 xylanases is very large in comparison with the few products generated by the same enzymes acting on chemically deacetylated polysaccharide. $^{27)}$ With exception of traces of non-acetylated Xyl and $\mathrm{Xyl}_{2}$, the acetylxylan hydrolysates do not contain other

A

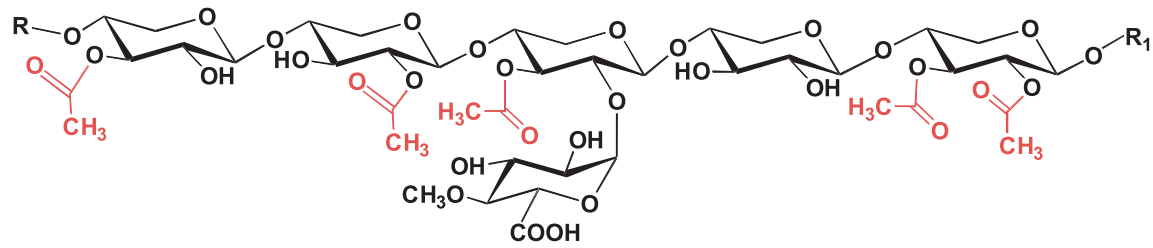

B

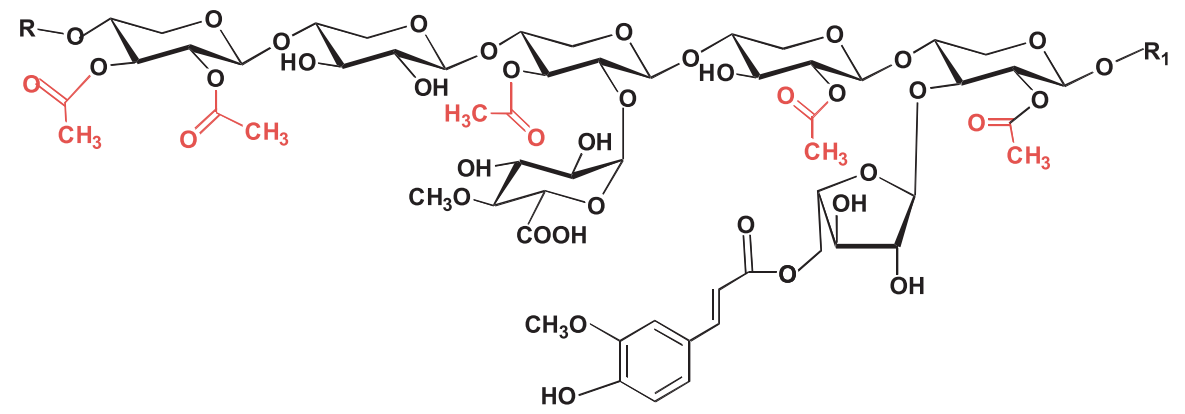

Fig. 1. Acetylation of xylosyl residues in hardwood acetylglucuronoxylan (A) and cereal or annual plant arabinoglucuronoxylan (B).

MeGlcA in xylan B can be replaced by GlcA. The acetylation of position 2 in Xylp residues 3-O-substituted with L-arabinose and esterified at position 5 with ferulic acid has been reported only recently in highly substituted corn fiber xylan. ${ }^{26)}$ Another structural feature of corn fiber xylan is substitution of Larabinose at position 2 by xylose residue or $2-O-\alpha$-galactosylated xylosyl residue. ${ }^{26}$ 
non-acetylated xylan fragments. These results highlight the important role of acetylxylan esterases (AcXEs) in microbial degradation of native acetylated polysaccharides. As expected, the GH10 xylanase produced shorter products than GH11 xylanase from both acetylated and deacetylated polysaccharide. Aldotetraouronic acid $\mathrm{MeGlc}^{3} \mathrm{Xyl}_{3}$ is the major acidic product of GH10 xylanase and aldopentaouronic acid $\mathrm{MeGlcA}^{3} \mathrm{Xyl}_{4}$ the major acidic product of $\mathrm{GH} 11$ xylanase acting on the deacetylated polysaccharide. Another difference showing the lower tolerance of the GH11 xylanases towards substitution by MeGlcA is the appearance of aldouronic acids with two residues of MeGlcA among the products of the GH11 xylanase hydrolysis (Biely and Westereng, unpublished data; see the bottom of Table 1). The crystal structure of GH10 xylanases with aldouronic ligands indicate that these require two consecutive unsubstituted Xyl $p$ residues for the cleavage of the xylanase main chain. ${ }^{28,29)}$ Several lines of evidence, particularly the analysis of the structure of products generated by GH11 xylanases, suggest that these enzymes require three consecutive unsubstituted Xyl $p$ residues. ${ }^{27)}$ It remains to be elucidated which of these Xylp residues could be acetylated. Exactly how acetyl groups affect the mode of cleavage of the xylan main chain remains unknown and little information is available on the tolerance of acetyl groups on the xylan main chain by appendage-dependent GH30 and GH5 xylanases (glucuronoxylan hydrolases and arabinoxylan hydrolases). ${ }^{30-33)}$

\section{DIVERSITY OF ACETYLXYLAN ESTERASES}

The variability of acetylation of Xylp residues (Fig. 1) poses various questions on the effective hydrolysis of the acetylated polysaccharide. For example, how many different AcXEs are needed to remove acetyl groups from all positions? Can complete deacetylation be achieved by a single enzyme in the absence of endoxylanases? Is the diversity of carbohydrate esterases linked also to variety of acetyl groups in acetylated xylans? According to the CAZy classification, ${ }^{34)}$ among the 16 carbohydrate esterase families we can identify characterized AcXEs in families 1, 3, 4, 5 and 6. Here we define AcXEs as only those enzymes that can deacetylate the polymeric substrate so efficiently as to cause it to precipitate from solution. ${ }^{5}$ )

AcXEs acting on acetylated polysaccharides which are too large to be transported into the cells, are likely to be extracellular, and maybe bound in bacterial cellulosomes ${ }^{35,36)}$ (the polymer is too large to be transported into the cell). The enzymes may also be intracellular, where they would act on soluble acetylated xylooligosaccharides imported into the cells. ${ }^{37-40)}$ A transport system for linear and branched arabinoxylooligosaccharides also occurs in the probiotic strain of Bifidobacter animalis subsp. lactis B1-04. ${ }^{41)}$ A xylobiose permease was earlier demonstrated as a part of the xylanolytic system of the yeast Cryptococcus albidus ${ }^{42)}$ and a Streptomyces strain. ${ }^{43}$ None of the above reports deals with acetylated xylooligosaccharides, but they are most probably transported by the same carriers as non-acetylated

Table 1. Neutral and acidic xylooligosaccharides generated from aspen acetylglucuronoxylan by GH10 (Clostridium thermocellum) and GH11 (Optimase, Genencor) endoxylanases as revealed by MALDI ToF MS (masses correspond to sodium adducts; for experimental details see Ref. 55).

\begin{tabular}{|c|c|c|c|c|c|}
\hline Compound & $\begin{array}{c}\text { Ions of neutral } \\
\text { oligosaccharides by } \\
\text { GH10 }\end{array}$ & $\begin{array}{c}\text { Ions of neutral } \\
\text { oligosaccharides by } \\
\text { GH11 }\end{array}$ & Compound & $\begin{array}{c}\text { Ions of acidic } \\
\text { oligosaccharides by } \\
\text { GH10 }\end{array}$ & $\begin{array}{c}\text { Ions of acidic } \\
\text { oligosaccharides by } \\
\text { GH11 }\end{array}$ \\
\hline $\mathrm{Xyl}_{2}$ & $(305)$ & & $\mathrm{MeGlcAXyl}_{2} \mathrm{Ac}$ & 537 & \\
\hline $\mathrm{Xyl}_{2} \mathrm{Ac}$ & 347 & & $\mathrm{MeGlcAXyl}_{3} \mathrm{Ac}$ & 669 & 669 \\
\hline $\mathrm{Xyl}_{2} \mathrm{Ac}_{2}$ & 389 & & $\mathrm{MeGlcAXYl}_{3} \mathrm{Ac}_{2}$ & 711 & \\
\hline $\mathrm{Xyl}_{3} \mathrm{Ac}$ & 479 & 479 & $\mathrm{MeGlcAXYl}_{3} \mathrm{Ac}_{3}$ & 753 & \\
\hline $\mathrm{Xyl}_{3} \mathrm{Ac}_{2}$ & 521 & & MeGlcAXyl 4 Ac & $(801)$ & $(801)$ \\
\hline $\mathrm{Xyl}_{3} \mathrm{Ac}_{3}$ & 563 & & $\mathrm{MeGlcAXyl}_{4} \mathrm{Ac}_{2}$ & 843 & 843 \\
\hline $\mathrm{Xyl}_{4} \mathrm{Ac}_{2}$ & 653 & 653 & $\mathrm{MeGlcAXyl}_{4} \mathrm{Ac}_{3}$ & 885 & 885 \\
\hline $\mathrm{Xyl}_{4} \mathrm{Ac}_{3}$ & 695 & 695 & $\mathrm{MeGlcAXyl}_{5} \mathrm{Ac}_{2}$ & $(975)$ & 975 \\
\hline $\mathrm{Xyl}_{4} \mathrm{Ac}_{4}$ & 737 & & $\mathrm{MeGlcAXyl}_{5} \mathrm{Ac}_{3}$ & 1017 & 1017 \\
\hline $\mathrm{Xyl}_{5} \mathrm{Ac}_{2}$ & 785 & 785 & $\mathrm{MeGlcAXyl}_{5} \mathrm{Ac}_{4}$ & 1059 & 1059 \\
\hline $\mathrm{Xyl}_{5} \mathrm{Ac}_{3}$ & 827 & 827 & MeGlcAXyl ${ }_{6} \mathrm{Ac}_{3}$ & 1149 & 1149 \\
\hline $\mathrm{Xyl}_{5} \mathrm{Ac}_{4}$ & 869 & 869 & $\mathrm{MeGlcAXyl}_{6} \mathrm{Ac}_{4}$ & 1191 & 1191 \\
\hline $\mathrm{Xyl}_{5} \mathrm{Ac}_{5}$ & 911 & & $\mathrm{MeGlcAXyl}_{6} \mathrm{Ac}_{5}$ & 1233 & 1233 \\
\hline $\mathrm{Xyl}_{6} \mathrm{Ac}_{3}$ & 959 & 959 & $\mathrm{MeGlcAXyl}_{7} \mathrm{Ac}_{4}$ & (1323) & 1323 \\
\hline $\mathrm{Xyl}_{6} \mathrm{Ac}_{4}$ & 1001 & 1001 & $\mathrm{MeGlcAXyl}_{7} \mathrm{Ac}_{5}$ & 1365 & 1365 \\
\hline $\mathrm{Xyl}_{6} \mathrm{Ac}_{5}$ & 1043 & 1043 & $\mathrm{MeGlcAX} \mathrm{l}_{7} \mathrm{Ac} 6$ & 1407 & 1407 \\
\hline $\mathrm{Xyl}_{6} \mathrm{Ac}_{6}$ & 1085 & 1085 & $\mathrm{MeGlcAXyl}_{8} \mathrm{Ac}_{4}$ & 1455 & 1455 \\
\hline $\mathrm{Xyl}_{7} \mathrm{Ac}_{4}$ & 1133 & 1133 & MeGlcAXyl 8 Acs $_{5}$ & 1497 & 1497 \\
\hline $\mathrm{Xyl}_{7} \mathrm{Ac}_{5}$ & 1175 & 1175 & MeGlcAXyl $8 \mathrm{Ac}_{6}$ & 1539 & 1539 \\
\hline $\mathrm{Xyl}_{7} \mathrm{Ac}_{6}$ & 1217 & & $\mathrm{MeGlcAX} \mathrm{Xl}_{8} \mathrm{Ac}_{7}$ & 1581 & 1581 \\
\hline $\mathrm{Xyl}_{7} \mathrm{Ac}_{7}$ & 1259 & & $\mathrm{MeGlcAXy}_{9} \mathrm{Ac}_{6}$ & 1671 & 1671 \\
\hline $\mathrm{Xyl}_{8} \mathrm{Ac}_{5}$ & & 1307 & $\mathrm{MeGlcAXyl}_{9} \mathrm{Ac}_{7}$ & 1713 & 1713 \\
\hline $\mathrm{Xyl}_{8} \mathrm{Ac}_{6}$ & & 1349 & MeGlcAXyloAc8 & 1755 & 1755 \\
\hline $\mathrm{Xyl}_{8} \mathrm{Ac}_{7}$ & & & $\mathrm{MeGlcA}_{2} \mathrm{Xyl}_{7} \mathrm{Ac}_{4} *$ & & 1513 \\
\hline $\mathrm{Xyl}_{8} \mathrm{Ac} 8$ & & & $\mathrm{MeGlcA}_{2} \mathrm{Xyl}_{7} \mathrm{Ac}_{5} *$ & & 1555 \\
\hline $\mathrm{Xyl}_{9} \mathrm{Ac}_{5}$ & & 1439 & $\mathrm{MeGlcA}_{2} \mathrm{Xyl}_{8} \mathrm{Ac}_{4} *$ & & 1645 \\
\hline $\mathrm{Xyl}_{9} \mathrm{Ac}_{6}$ & & 1481 & $\mathrm{MeGlcA}_{2} \mathrm{Xyl}_{8} \mathrm{Ac}_{5} *$ & & 1687 \\
\hline
\end{tabular}

Acidic oligosaccharides containing two MeGlcA residues are marked by asterisks. Xyl and $\mathrm{Xyl}_{2}$ are the only deacetylated products observed in small quantities in the enzymatic hydrolysates of acetylglucuronoxylan. 


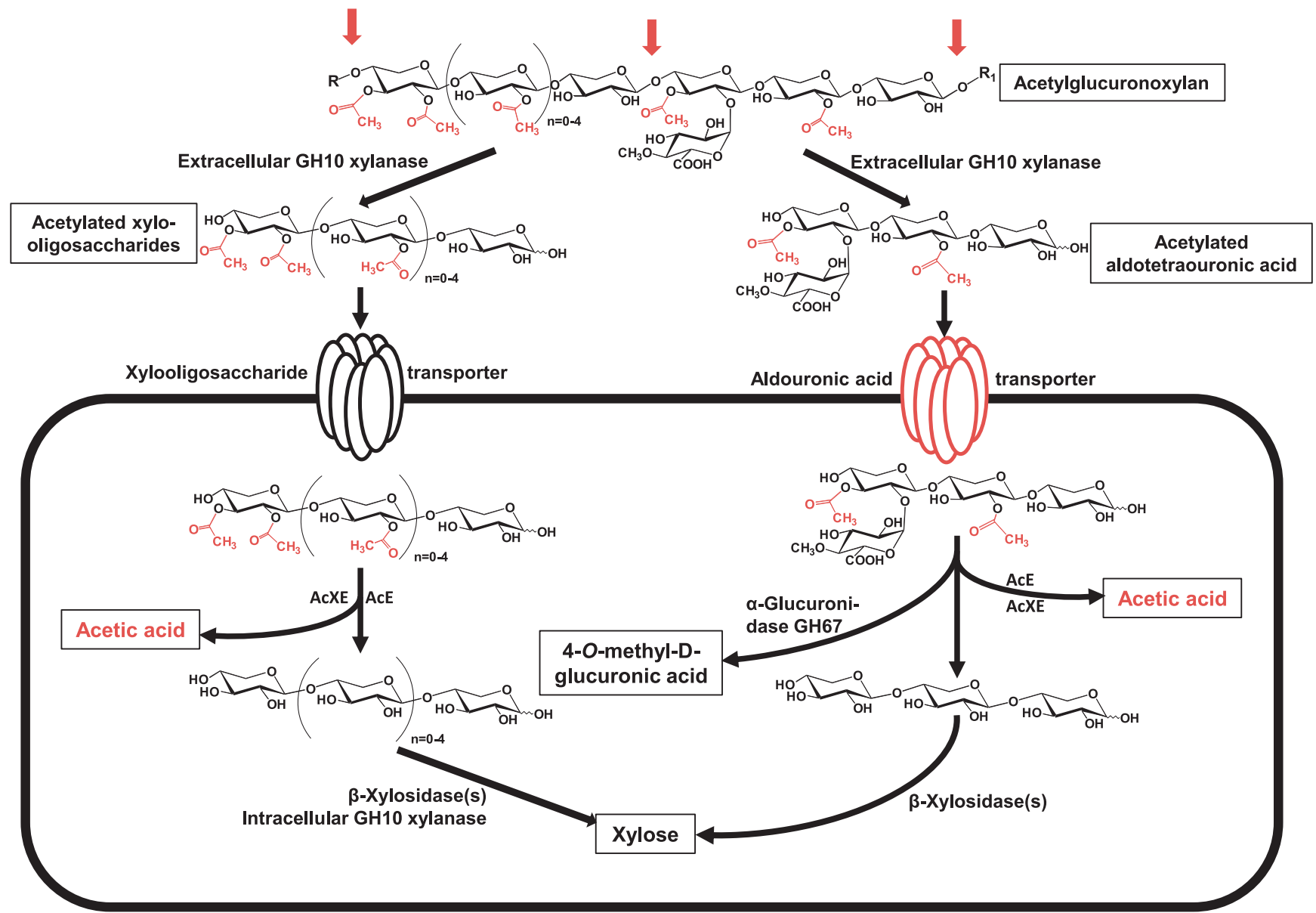

Fig. 2. Proposed model for intracellular breakdown of xylooligosaccharides in some bacteria.

The acetylglucuronoxylan is cleaved extracellularly into acetylated xylooligosaccharides and aldotetrauronic acid, which are internalized by specific oligosaccharide transport systems. Subsequently these oligosaccharides are deacetylated and hydrolyzed to monosaccharides by intracellular esterases and glycoside hydrolases. Based on Refs. 37-40) and 44).

compounds. Once in the cell, acetylated oligosaccharides would be substrates for intracellular esterases. ${ }^{40,44}$ Candidate for this process would be esterases of CE family 7. This family includes intracellular acetylesterases which do not recognize large polymeric substrates but deacetylate glycosides (alkyl and aryl $\beta$-xylopyranosides) and oligosaccharides. ${ }^{45-47)}$ A model for intracellular breakdown of extracellularly generated acetylated xylooligosaccharides that involves the interplay of intracellular deacetylases and glycosidases can be deduced from the genomic and biochemical evidence from a number of bacterial species (Fig. 2) ${ }^{37-40,44)}$

\section{MODE OF ACTION OF AcXEs}

The positional specificity of AcXEs was earlier studied using artificial substrates; mono- or di-O-acetylated methyl $\beta$-xylopyranoside, their deoxy- and deoxy-fluoroderivatives, 4 -nitrophenyl $\beta$-xylopyranoside and glycosides of hexoses (reviewed in Ref. 5). However, the use of these substrates did not provide unequivocal guidelines for specificity. Several AcXEs deacetylated methyl $\beta$-xylopyranoside at position 2 and 3 , in contrast to their strong preference for position 2 in monoacetyl derivatives of 4-nitrophenyl $\beta$-xylopyranoside. ${ }^{48}$ A study on the mode of action of several AcXEs on partially acetylated cellulose showed that some AcXEs exhibited regioselectivity, while others did not. ${ }^{49)}$ The data obtained with deoxy and deoxy-fluoro-analogues of 2- or 3-monoacetylated methyl $\beta$-xylopyranoside showed major differences in specificities between serine-type esterases belonging to carbohydrate esterase families 1 and 5, and AcXEs of family CE4, the aspartate metalloenzymes. ${ }^{5)}$ The CE4 AcXEs required the vicinal OH-group free for deacetylation of position 2 or 3 in Xylp residues, such that 2,3 -di- $O$-acetyl or fully acetylated methyl $\beta$-D-xylopyranoside did not serve as substrates. ${ }^{50-52)}$

The evaluation of the total amount of released acetyl groups from a mixture of acetylated xylooligosaccharides by two AcXEs (CE1 and CE5 family) from Chrysosporium lucknowense $\mathrm{C} 1$ (renamed as Myceliophtora thermophila $\left.\mathrm{C}^{53}\right)$ suggested that the enzymes deacetylate both positions 2 and 3, which was in accord once with the mode of action of enzymes on acetylated methyl xylopyranosides but in contradiction with the data observed on monoacetyl derivatives of 4-nitrophenyl $\beta$-D-xylopyranoside. ${ }^{48)}$ These observations stimulated a more detailed analysis of the mode of action of AcXEs on native, partially acetylated glucuronoxylan extracted from hardwood using ${ }^{1} \mathrm{H}-\mathrm{NMR}$ spectroscopy. The complete assignment of the proton resonances of all acetyl groups in the polysaccharide, both of resonances of esterified Xylp residues and those of the methyl protons of the acetyl groups was achieved. ${ }^{54}$ This approach was used to follow the positional specificity and mode of action of AcXEs in deuterized buffer by observing the decrease in the intensity of the signals of particular acetyl groups. Members 
of the following CE families were examined: Schizophyllum commune CE1 AcXE, Streptomyces lividans and Clostridium thermocellum CE4 AcXEs and Orpinomyces CE6 AcXE. ${ }^{54,55)}$ All enzymes tested were found to de-esterify $\mathrm{Xyl} p$ residues monoacetylated at both positions 2 and 3 . The major difference found between the modes of action of serine type AcXEs belonging to CE families 1, 5 and 6 and aspartate metallo-enzymes of CE4 family, was the inability of the latter to deesterify doubly acetylated Xylp residues. This is apparently the consequence of the requirement for a free vicinal OH-group which participates in the formation of the enzyme-substrate complex by coordinating with the catalytic metal ion. This interaction appears to be indispensable for deacetylation of the vicinal position, regardless whether it is position 2 or $3{ }^{56)}$ None of the enzymes were capable of attacking the 3-O-acetyl group on the Xylp residue substituted with MeGlcA residue. However, for serine-type esterases attacking diacetylated Xyl $p$ residues, which do not require a vicinal hydroxyl group, the MeGlcA residue in position 2 is a much larger substituent than the acetyl group, and is likely to represent a serious steric hindrance to hydrolyze the neighbouring 3-O-acetyl group.

The ${ }^{1} \mathrm{H}-\mathrm{NMR}$ studies of the mode of action of AcXEs confirmed the capability of members of four different CE families to catalyze deacetylation of internal Xylp residues in the polymeric substrate. ${ }^{21,55}$ It is probable that some of the enzymes recognize several Xyl $p$ residues of the main chain as is typical for the glycoside hydrolases. Examples are found in chitin deacetylases, where the 3D structures show substrate binding sites comprising several subsites binding $N$-acetyl-D-glucosaminyl residues. ${ }^{10)}$ The deacetylation of hexopyranosides $^{57)}$ and cellulose acetate by $\mathrm{AcXEs}^{49)}$ suggests that the enzymes tolerate the replacement of Xyl $p$ residues by hexopyranosyl residues. This is consistent with the observation that CE4 AcXEs catalyze $N$-deacetylation of chitin, an example of their action on acetylated hexopyranosyl residues. ${ }^{56,58)}$

One of the most striking observations from studies of the positional specificity of AcXEs is the finding that both serine- and aspartate-type AcXEs (the CE4 family enzymes) deacetylate monoacetylated Xyl $p$ residues at positions 2 and 3 , earlier observed with methyl $\beta$-D-xylopyranosides. ${ }^{5}$ It was originally proposed that the deacetylation of the two positions involved acetyl group migration in the active site of the enzyme. ${ }^{5)}$ However this concept lost favour after the 3D structure of T. reesei AcXE was elucidated. ${ }^{59)}$ The authors suggested that the 2- or 3-acetylated methyl $\beta$-D-xylopyranoside could form two different $180^{\circ}$ oriented enzymesubstrate complexes ${ }^{59)}$ with identical stereochemical arrangements of the hydroxyl groups. ${ }^{5)}$ While this model is clearly feasible for small substrates, it is not readily compatible for polymeric substrates. A strong argument against the formation of two alternative $180^{\circ}$ flipped enzyme-substrate complexes is the fact that deacetylation of positions 2 and 3 is also catalyzed by CE4 AcXEs which comprise the same protein fold topology as chitin deacetylases, but whose active site structures are not homologous to that of $T$. reesei AcXE.

The hypothesis that deacetylation of both positions 2 and 3 is facilitated by acetyl group migration between the hydroxyls $\mathrm{OH}-2$ and $\mathrm{OH}-3$ has also been weakened by recent structural studies of GH62 $\alpha$-L-arabinofuranosidases, which are capable of removing both $\alpha-1,2-$ and $\alpha-1,3$-linked L-arabinofuranosyl side residues from the xylan main chain. ${ }^{60,61)}$ The studies demonstrate that these enzymes form two oppositely oriented productive complexes with the polymeric substrate, ${ }^{60,61)}$ as was once proposed for AcXEs. ${ }^{59)}$

\section{DEACETYLATION OF XYLOOLIGOSACCHARIDES BY AcXES}

It has been clearly demonstrated that AcXEs and endoxylanases act on acetyl xylan synergistically; i.e. xylanases stimulate the rate of deacetylation and AcXE stimulate the rate of hydrolysis of glycosidic linkages. ${ }^{62-65)}$ The AcXEs create new sites on the xylan main chain for productive binding with endoxylanases. Conversely, cleavage of the main chain exposes new sites for deacetylation. The depolymerization of the polysaccharide may also have additional positive effects on the rate of substrate deacetylation. Decreasing the size of the substrate increases its diffusion rate and thus the rate of deacetylation.

The effect of AcXEs belonging to CE families 1, 4, 5 and 6 on aspen acetylglucuronoxylan hydrolysis products generated by the action of GH10 from Clostridium thermocellum (CtGH10), was elucidated by MALDI ToF MS. $\left.{ }^{55}\right)$ This method allowed a detailed comparison of the oligosaccharides before and after AcXE treatment.

The deacetylation of oligosaccharides by all AcXEs tested was consistent with their performance on polysaccharides. For both neutral and acidic oligosaccharides, CE4 AcXEs hydrolyzed one or two acetyl group less than the serine-type AcXEs. While serine-type esterases deacetylated neutral oligosaccharides either completely or to monoacetates, the CE4 AcXEs left a considerable portion of the products also in the form of diacetates. Similarly, while the serine type esterases converted acidic oligosaccharides either to the deacetylated or the mono- and di-O-acetate forms, the products of CE4 AcXE activity included derivatives with three acetyl groups. ${ }^{55}$ ) This difference is obviously the consequence of the inability of the CE4 AcXEs to attack doubly acetylated Xylp residues. Incomplete deacetylation by AcXEs of CE families 1 and 5 was also observed with xylooligosaccharides derived from the eucalyptus wood ${ }^{60}$ where a considerable fraction of both neutral and acidic xylooligosaccharides remained monoacetylated. The exact mode of action of AcXEs on polymeric substrate ${ }^{54,55}$ has yet to be clarified, however, based on the mode of action of AcXEs on artificial substrates like monoacetates of 4-nitrophenyl $\beta$-xylopyranosides, ${ }^{48}$ it can hypothesized that the monoacetates of neutral xylooligosaccharides carry single acetyl group at position 4, as a result of acetyl group migration. In the monoacetates of the acidic xylooligosaccharides generated by GH10 endoxylanase, i.e. aldouronic acids with $\alpha-1,2$-linked MeGlcA residues, the nonreducing $\mathrm{Xyl} p$ residue may have the single acetyl group either at the original position 3 or at position 4 as a result of acetyl group migration from position 3. In the case of CE4 AcXEs, diand tri- $O$-acetates are also observed to be resistant to enzymatic hydrolysis. The proposed structures of xylooligosaccharide mono- di- and triacetates resistant to serine-type and aspartate-type AcXEs are depicted in Fig. 3. 
A

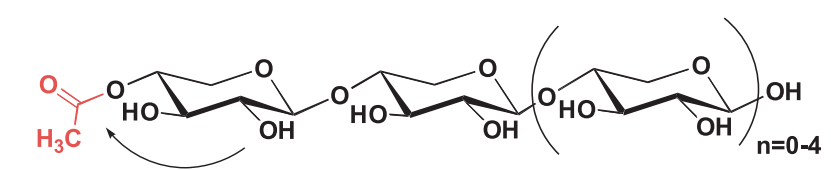

B

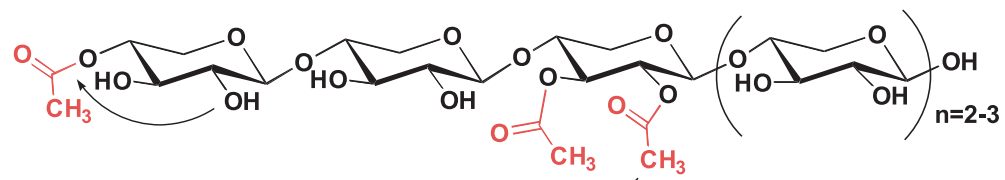

C

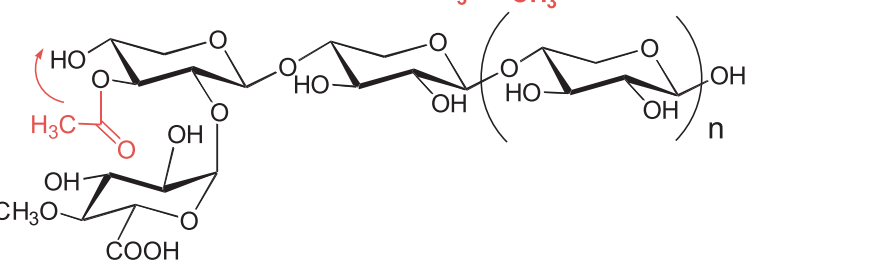

D

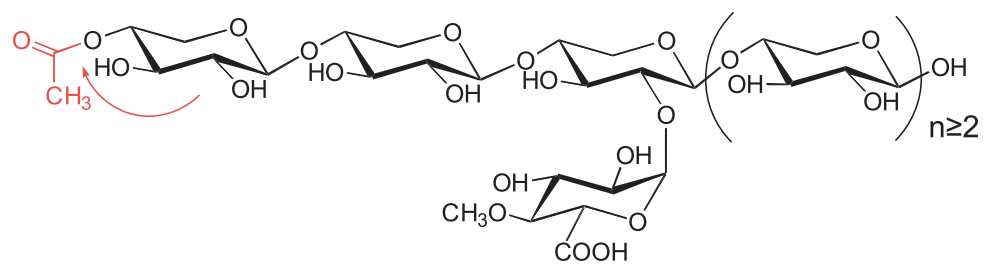

$E$

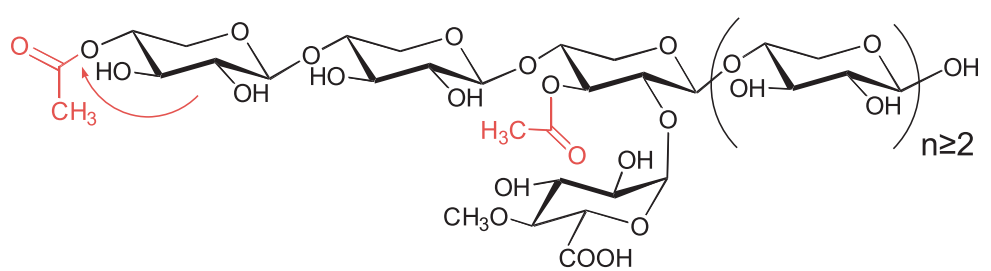

$\mathrm{F}$

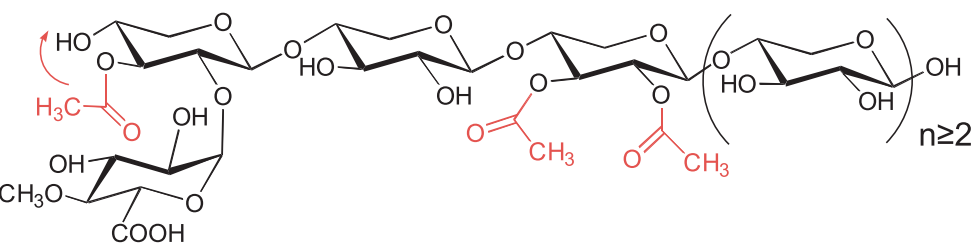

Fig. 3. Suggested structural features of acetylated neutral (A, B) and acidic oligosaccharides (C-D) in the hydrolysate of acetylglucuronoxylan with GH10 xylanase resistant to AcXEs (GH10 xylanase was denatured before the AcXE treatment).

With exception of the structures $\mathrm{C}$ and $\mathrm{F}$, all xylooligosaccharides are shown acetylated in position 4 of the non-reducing Xylp residue, where the acetyl group migrated (thin red arrows). The 4-acetate is in the quilibrium with the 3-and 2-O-acetyl derivatives in linear xylooligosaccharides (A and B) and with 3-O-acetylated derivative in aldouronic acids carrying MeGlcA residue on the non-reducing end ( $\mathrm{C}$ and $\mathrm{F})$. The internal 2,3-di-O-acetylated Xylp residues (B and F) are resistant only to CE4 AcXEs. Based on Refs. 55, 66) and 71).

\section{ACETYL GROUP MIGRATION TO POSITION 4}

The proposal for the resistance of the 4-acetyl group to the action of AcXEs is based, in part, on kinetic measurements of the migration of the acetyl group in monoacetates and diacetates of 4-nitrophenyl $\beta$-D-xylopyranosides. ${ }^{67)}$ At equilibrium, the 4-O-acetyl derivative predominates in monoacetates and the 3,4-derivative in diacetates. The 2-O-acetyl derivatives are the most kinetically labile. Acetyl group migration was assessed at $40^{\circ} \mathrm{C},{ }^{67)}$ a temperature much lower than used in many non-alkaline hydrothermal pretreatments of lignocellulose biomass. Examination of the rate of acetyl group migration on monoacetates of 4-nitrophenyl $\beta$-xylopyranoside at $100^{\circ} \mathrm{C}$ confirmed that the positional isomer equilibrium is established very rapidly (within a few minutes, even at $\mathrm{pH}<7$; M. Cziszárová and $\mathrm{P}$. Biely, unpublished data). Acetyl migration has also been observed for monoacetyl derivatives of methyl $\beta$-D-xylotrioside prepared by $T$. reesei $\mathrm{CE} 16$ acetylesterase transacetylation. ${ }^{68)}$ The enzyme transfers the acetyl group exclusively to the $\mathrm{OH}-3$ of the non-reducing Xylp residue, although a single reaction product cannot be isolated due to migration of the acetyl group from position 3 .

Other data supporting the resistance of the 4-acetyl group at the non-reducing terminal to the action of AcXEs include the relative rates of deacetylation of the monoacetates of 4-nitrophenyl $\beta$-xylopyranoside by AcXEs ${ }^{48}$ and the regioselectivity of deacetylation of per- $O$-acetylated glycosides. ${ }^{57)}$ AcXEs show negligible or extremely low activity on the acetyl group at position 4 of a variety of acetylated glycosides, but deacetylate rapidly positions 2 and $3 .{ }^{57)}$ Treatment of the equilibrium mixture of three monoacetyl 
derivatives of methyl $\beta$-D-xylotrioside by AcXEs belonging to $\mathrm{CE}$ families 1, 4 and 6 clearly confirmed that the $4-O$-acetyl derivative is the most resistant, although a slow but significant hydrolysis was observed with the CE1 AcXE (V. Puchart and P. Biely, unpublished data).

On the basis of these studies we conclude that any heat treatment of xylooligosaccharides acetylated at the non-reducing end, such as those extracted under high temperatures or generated from endoxylanase digestion of acetylated polysaccharide, will rapidly generate 4-Oacetylated derivatives which are not susceptible to deacetylation by AcXEs. However, since the 4-acetyl derivative are in equilibrium with 2-O- and 3-O-acetyl compounds, which are viable AcXE substrates, over long incubation period, the 4-acetyl derivatives will slowly be removed.

\section{ENZYMES DEACETYLATING NON-REDUCING ENDS}

Enzymes deacetylating the non-reducing end of oligosaccharides are found in CE family16. The first enzyme identified with this specificity was the acetylesterase of $T$. reesei classified as the first member of the CE family $16 .{ }^{69,70)}$ The enzyme did not deacetylate polymeric substrates but acted on acetylated xylobiose ${ }^{69)}$ On monoacetyl derivatives of 4-nitrophenyl $\beta$-D-xylopyranoside the enzyme showed preference for deacetylation of position 3 and $4,{ }^{48}$ ) with specific activities for the 2-acetate approximately 20 times lower. The CE16 esterase also efficiently catalyzed transacetylation to position 3 of the non-reducing ends of cello-, manno- and xylooligosaccharides ${ }^{68)}$ with removal of the acetyl in the absence of an acetyl donor. ${ }^{68)}$ Based on these catalytic properties the enzyme was assigned as an exo-deacetylase, ${ }^{5}$ and exo-activity was subsequently confirmed on acetylated xylooligosaccharides using MALDI ToF MS. ${ }^{66,71)}$ The application of this enzyme with AcXEs leads to almost complete deacetylation of neutral and acidic xylooligosaccharides. When applied as the sole enzyme, the CE16 esterase typically removes only one acetyl group. A similar specificity is observed with MeGlcA-substituted non-reducing ends of aldouronic acids, although evidence whether the deacetylation takes place at position 3 or 4 , or both is currently lacking. The migration of the acetyl group to position 4 would move the acetyl group away from the MeGlcA, thereby potentially reducing steric inhibition. Support for this suggestion was obtained by testing the enzyme on di-O-acetates and tri- $O$-acetate of methyl $\beta$-D-xylopyranoside at $10 \mathrm{mM}$ substrate concentration. ${ }^{71)}$ The enzyme showed a preference for deacetylation of position 3 in 2,3-diacetate. The 2,4-diacetate was deacetylated about 5-fold slower at position 4 and the 3,4-diacetate about 2 to 3 orders of magnitude slower. The fully acetylated glycoside was resistant to the enzyme. ${ }^{71)}$ These observations suggest that for the deacetylation of position 3 substituents at position 2 show the lowest steric influence.

A higher degree of xylooligosaccharide deacetylation was reported with the CE16 acetyl esterase from M. thermophila $\mathrm{C} 1{ }^{66}{ }^{66}$ only the second CE16 esterase investigated in this regard. In the presence of a GH family 10 endoxylanase and a $\beta$-xylosidase, the enzyme released $79 \%$ of acetic acid from a mixture of neutral and acidic acetylated xylooligosaccha- rides. Combination with T. reesei AcXE (CE5) led to 88\% deacetylation. Addition of GH115 $\alpha$-glucuronidase to the enzyme mixture not only resulted in almost complete release of MeGlcA but also in near quantitative release of acetic acid. ${ }^{66)}$

Naturally, the migration of the acetyl group to position 4 may not be of such significance in nature where the acetylated xylan is attacked simultaneously with a mixture of endoxylanases, esterases and accessory xylanolytic enzymes. The acetyl group at the non-reducing end of oligosaccharides generated by endoxylanases and eventually $\beta$-xylosidase would be primarily located at position 2 or 3 where it could immediately be attacked by AcXEs and CE16 acetylesterase. Debranching of the main xylan chain or oligosaccharides by $\alpha$-glucuronidase and $\alpha$-arabinofuranosidase would proceed synergistically with simultaneous deacetylation.

\section{CONCLUDING REMARKS AND OUTLOOK}

The importance of the participation of deacetylating carbohydrate esterases in the breakdown of native partially acetylated plant hemicelluloses by microorganisms is clear, where the esterases appear to work synergistically with glycoside hydrolases. The high chemical and structural complexity of plant hemicelluloses appears to be balanced in nature by a very high diversity of degradative enzymes differing in the modes of action and substrate specificities. Here we have reviewed the recent progress in our knowledge of a specific group of esterases which participate in the degradation of partially acetylated plant xylans. The AcXEs are unique enzymes harbouring two structurally diverse groups; the aspartate metalloenzymes and serine-type enzymes with differing capabilities to deacetylate doubly acetylated Xyl $p$ residues. One of the most interesting questions posed from recent studies is the mechanistic basis of AcXE deacetylation at positions 2 and 3 in the polysaccharide. A deeper insight into the active site structure of the esterases will be needed to clarify this "positional" non-specificity.

Microorganisms also produce specialized esterases capable of removing acetyl groups not readily accessible to AcXEs. An important recent contribution to this area is a partial recognition of the role of CE16 acetylesterases, which effectively deacetylate positions which do not occur in native polysaccharide. ${ }^{5,66,71)} \mathrm{We}$ refer in particular to the acetyl group at position 4 of non-reducing Xylp residues, where this group migrates rapidly during heat treatment of the acetylated oligosaccharides. This is an important issue for the development of processes for the complete saccharification of acetylated xylan. In nature, where the polymer is attacked simultaneously by a consortium of glycoside hydrolases and esterases the 4-acetylation at the non-reducing end may not be of such significance.

None of the members of the CE16 acetylesterases has been crystallized to date. Acquisition of a high resolution 3D structure will be particularly important in understanding the interaction of the enzyme with MeGlcA substituted and 3-O-acetylated non-reducing Xyl $p$ residues as well as resolving the mechanism of the activation of the CE16 enzyme with carbohydrates when acting on non-carbohydrate substrates. ${ }^{70)}$ 
We should emphasize that all new data on the mode of action of AcXEs and of CE16 esterases on xylooligoaccharides comes from the experiments with hydrolysis products generated by family 10 endoxylanase, which cleaves the acetylglucuronoxylan main chain at the glycosidic linkage adjacent to 3-O-acetylated and by MeGlcA- or GlcA-substituted Xyl $p$ residues. ${ }^{27-29)}$ Similar studies should be carried out with acetylated xylooligosaccharides generated by other types of endoxylanases, including the recently discovered appendage-dependent xylanases of family GH30 and GH5. $\mathrm{GH}$ family 11 xylanase would most probably generate aldouronic acids with MeGlcA-substituted and 3-O-acetylated $\mathrm{Xyl} p$ residues on the penultimate residue from the non-reducing end. The 3-O-acetyl group could become the substrate of CE16 acetyesterases only after removal of the terminal xylose by $\beta$-xylosidase.

Whether polymer-attacking enzymes deacetylating position 3 in internal Xyl $p$ residues substituted with MeGlcA or acetyl group at position 2 in $\mathrm{Xyl} p$ residues substituted by L-Ara occurring in cereal arabinoxylans ${ }^{26)}$ exist in nature, remains to be investigated.

There are numerous esterases reported in the literature which have not been examined for their abilities to deacetylate hemicellulose-derived carbohydrates. Many microorganisms utilize xylan as a carbon sourse, but do not degrade xylan fragments extracellularly. These oligosaccharides are transported into the cells by inducible transport systems and fully degraded intracellularly. Evidence has been presented that there are transport systems for both neutral and acidic oligosaccharides, ${ }^{37-40)}$ but it is not known whether these systems also translocate acetylated xylooligosaccharides. Some intracellular acetylesterases, like those of the CE7 family ${ }^{44-47)}$ appear to have very wide substrate specificity, potentially offering a variety of useful applications.

Recent attempts to reduce the degree of acetylation of plant cell wall polysaccharides by genetic modifications, with the aim of making them more accessible to degradation by glycoside hydrolases and to reduce the acetate-mediated inhibition of fermentations, have clearly demonstrated that acetylation of hemicelluloses is inevitable for plants and that the reduction of the acetyl groups content in plant polysaccharides may not lead to expected results. ${ }^{6-8)}$ Suggestions to genetically modify plant hemicellulose structure by replacing the acetyl group side residues by fermentable sugars ${ }^{7)}$ is an exciting prospect for future research.

\section{ACKNOWLEDGMENTS}

This work was supported by grants from the Slovak Academy of Sciences grant agency VEGA 2/0037/14, by The Slovak Research and Development Agency under the contract No. APVV-0602-12, by grant 214613 from the Norwegian Research Council and by the FP7 project Waste2Go under contract 308363 with European Commission. DAC acknowledges support from the University of Pretoria Genomic Research Institute. PB gratefully acknowledges receipt of a University of Pretoria Visiting Professor Award.

\section{REFERENCES}

1 ) S. Gille and M. Pauly: $O$-Acetylation of plant polysaccharides. Front. Plant Sci., 3, 12 (2012).

2 ) P.M.-A. Pawar, S. Koutaniemi, M. Tenkanen and E.J. Mellerowicz: Acetylation of woody lignocelluloses: significance and regula- tion. Front. Plant Sci., 4, 118 (2013).

3 ) T. Ishii: Structure and functions of feruloylated polysaccharides. Plant Sci., 127, 111-127 (1997).

4 ) G. Williamson, P.A. Kroon and C.B. Faulds: Hairy plant polysaccharides: a close shave with microbial esterases. Microbiology, 144, 2011-2023 (1998).

5 ) P. Biely: Microbial carbohydrate esterases deacetylating plant polysaccharides. Biotechnol. Adv., 30, 1575-1588 (2012)

$6)$ Y. Manabe, M. Nafisi, Y. Verhertbruggen, C. Orfila, S. Gille, C. Rautengarden, C. Cherk, S.E. Marcus, S. Somerville, M. Pauly, J.P. Knox, Y. Sakuragi and H.V. Scheller: Loss-of-function mutation of reduced wall acetylation2 in Arabidopsis leads to reduced cell wall acetylation and increased resistance to Botritis cinerea. Plant Physiol., 155, 1068-1078 (2011).

7 ) G. Xiong, K. Cheng and M. Pauly: Xylan $O$-acetylation impacts xylem development and enzymatic recalcitrance as indicated by the Arabidopsis mutant tbl29. Mol. Plant, 6, 1373-1375 (2013)

8 ) Y. Manabe, Y. Verhertbruggen, S. Gille, J. Harholt, S.-L. Chong, P.M.-A. Pawar, E.J. Mellerowicz. M. Tenkanen, K. Cheng, M. Pauly and H.V. Scheller: Reduced wall acetylation proteins play vital and distinct roles in cell wall $O$-acetylation in Arabidopsis. Plant Physiol., 155, 1107-1117 (2013).

9 ) K. Tokuyasu, M. Mitsutomi, I. Yamaguchi, K. Hayachi and Y. Mori: Recognition of chitooligosaccharides and their $N$-acetyl groups by putative subsites of chitin deacetylase from a deuteromycete, Clostridium lindemuthianum. Biochemistry, 39, 8837$8843(2000)$.

10) O. Hekmat, K. Tokuyasu and S.G. Withers: Subsite structure of the endo-type chitin deacetylase from a deuromycete, Clostridium lindemuthianum. An investigation using steady-state kinetic analysis and MS. Biochem. J., 374, 369-380 (2003).

11) C. Montanier, V.A. Money, V.M.R. Pires, J.E. Flint, B.A. Pinheiro, A. Goyal A, J.A.M. Prates, A. Izumi, H. Stälbrand, C. Morland, A. Cartmell, K. Kolenová, E. Topakas, E.J. Dodson, D.N. Bolam, G.J. Davies, C.M.G.A. Fontes and H.J. Gilbert: The active site of a carbohydrate esterase displays divergent catalytic and non-catalytic binding functions. PLoS Biol., 7, 687-697 (2009).

12) C.E. Wyman, B.E. Dale, R.T. Elander, M. Holzapple, M.R. Ladisch and Y.Y. Lee: Coordinated development of leading biomass pretreatment technologies. Bioresour. Technol., 96, 1959-1966 (2005).

13) M.J. Selig, E.P. Knoshaug, W.S. Adney, M.E. Himmel and S.R. Decker: Synergistic enhancement of cellobiohydrolase performance on pretreated corn stover by addition of xylanase and esterase activities. Bioresour. Technol., 99, 4997-5005 (2005).

14) S. Makishima, M. Mizuno, N. Sato, K. Shinji, M. Suzuki, K. Nozaki, F. Takahashi, T. Kanda and Y. Amano: Development of continuous flow type hydrothermal reactor for hemicelluloses fraction recovery from corncob. Bioresour. Technol., 100, 28422848 (2009).

15) P. Alvira, E. Tomás-Pejó, M. Ballesteros and M.J. Negro: Pretreatment technologies for an efficient bioethanol production process based on enzymatic hydrolysis: A review. Bioresour. Technol., 101, 4951-4861 (2010).

16) C.J. Barr, J.A. Mertens and C.A. Schall: Critical cellulose and hemicelluloses activities for hydrolysis of ionic liquid pretreated biomass. Bioresour. Technol., 104, 480-485 (2012).

17) S.H. Mood, A.H. Golfeshan, M. Tabatabaei, G.S. Jouzani, G.H. Najafi, M. Gholami and M. Ardjmand: Lignocellulosic biomass to bioethanol, a comprehensive review with a focus on pretreatment. Renew. Sustain. Ener. Rev., 27, 77-93 (2013).

18) A. Teleman, J. Lundqvist, F. Tjerneld, H. Stålbrand and O. Dahlman: Characterization of acetylated 4-O-methylglucuronoxylan isolated from aspen employing ${ }^{1} \mathrm{H}$ and ${ }^{13} \mathrm{C}$ NMR spectroscopy. Carbohydr. Res., 329, 807-815 (2000).

19) D. Evtuguin, J. Tomás, A.S. Silva and C. Neto: Characterization of an acetylated heteroxylan from Eucalyptus globulus Labill. Carbohydr. Res., 338, 597-604 (2003).

20) R. Naran, S. Black, S.R. Decke and P. Azadi: Extraction and characterization of native heteroxylans from delignified corn stover and aspen. Cellulose (Dordrecht, Neth.), 16, 661-675 (2009).

21) I. Uhliariková, M. Vršanská, B.V. McCleary and P. Biely: Positional specifity of acetylxylan esterases on natural polysac- 
charide: An NMR study. Biochim. Biophys. Acta, 1830, 33653372 (2013).

22) S. Dammstrom, L. Salmen and P. Gatenholm: On the interactions between cellulose and xylan, a biomimetic simulation of the hardwood cell wall. Bioresources, 4, 3-14 (2009).

23) S.L. Chong, T. Nissila, R.A. Ketola, S. Koutaniemi, M. DerbaMaceluch, E.J. Mellerowicz, M. Tenkanen and P. Tuomainen: Feasibility of using atmospheric pressure matrix-assisted laser desorption/ionization with ion trap mass spectrometry in the analysis of acetylated xylooligosaccharides derived from hardwoods and Arabidopsis thaliana. Anal. Bioanal. Chem., 401, 2995-3009 (2011)

24) Y. Yuan, Q. Teng, R. Zhong and Z.-H. Ye: The Arabidopsis DUF231 domain-containing protein ESK1 mediates 2-O- and 3-O-acetylation of xylosyl residues in xylan. Plant Cell Physiol., 54, 1186-1199 (2013).

25) R.B. Hespell: Extraction and characterization of hemicellulose from the corn fiber produced by corn wet-milling processes. $J$. Agric. Food Chem., 46, 2615-2619 (1998).

26) M.M. Appeldoorn, P. De Waard, M.A. Kabel, H. Gruppen and H.A. Schols: Enzyme resistant feruloylated xylooligomer analogues from thermochemically treated con fiber contain large side chains, ethyl glycosides and novel site of acetylation. Carbohydr. Res., 381, 33-42 (2013).

27) P. Biely, M. Vršanská, M. Tenkanen and D. Kluepfel: Endo- $\beta-1,4-$ xylanase families: differences in catalytic properties. J. Biotechnol., 57, 151-166 (1997).

28) G. Pell, E.J. Taylor, T.M. Gloster, J.P. Turkenburg, C.M.G.A. Fontes, L.M.A. Ferreira, T. Nagy, S.J. Clark, G.J. Davies and H.J. Gilbert: The mechanism by which family 10 glycoside hydrolases bind decorated substrates. J. Biol. Chem., 279, 9597-9605 (2004).

29) Z. Fujimoto, S. Kaneko, A. Kuno, H. Kobayashi, I. Kusakabe and H. Mizuni: Crystal structures of decorated xylooligosaccharides bound to a family 10 xylanase from Streptomyces olivaceoviridis E-86. J. Biol. Chem., 279, 9606-9614 (2004).

30) F.J. St John, J.D. Rice and J.F. Preston: Characterization of XynC from Bacillus subtilis subsp subtilis strain 168 and analysis of its role in depolymerisation of glucuronoxylan. J. Bacteriol., 188, 8617-8626 (2006).

31) M. Vršanská, K. Kolenová, V. Puchart and P. Biely: Mode of action of glycoside hydrolase family 5 glucuronoxylan xylanohydrolase from Erwinia chrysanthemi. FEBS J., 274, 1666-1677 (2007).

32) O. Gallardo, M. Fernández-Fernández, C. Valls, S.V. Valenzuela, M.B. Roncero, T. Vidal, P. Díaz and F.I.J. Pastor: Characterization of a family GH5 xylanase with activity on neutral oligosaccharides and evaluation as a pulp bleaching aid. Appl. Environ. Microbiol., 76, 6290-6294 (2010).

33) M.A.S. Correia, K. Mazumder, J.L.A. Bras, S.J. Firbank, Y.P. Zhu, R.J. Lewis, W.S. York, C.M.G.A. Fontes and H.J. Gilbert: Structure and function of an arabinoxylan-specific xylanase. $J$. Biol. Chem., 286, 22510-22520 (2011).

34) V. Lombard, H.G. Ramulu, E. Drula, P.M. Coutinho and B. Henrissat: The carbohydrate-active enzymes database (CAZy) in 2013. Nucleic Acids Res., 42, D490-D495 (2014).

35) E.A. Bayer, J.P. Belaich, Y. Shoham and R. Lamed: The cellulosomes: multienzyme machines for degradation of plant cell wall polysaccharides. Annu. Rev. Microbiol., 58, 521-554 (2004).

36) C.M.G.A. Fontes and H.J. Gilbert: Cellulosomes: highly efficient nanomachines designed to deconstruct plant cell wall complex carbohydrates. Annu. Rev. Biochem., 79, 655-681 (2010).

37) S. Shulami, G. Zaide, G. Zolotnitsky, Y. Langut, G. Feld, A.L. Sonenshein and Y. Shoham: A two-component system regulates the expression of an $\mathrm{ABC}$ transporter for xylo-oligosaccharides in Geobacillus stearothermophilus. Appl. Environ. Microbiol., 73, 874-884 (2007).

38) V. Chow, G. Nong and J.F. Preston: Structure, function, and regulation of the aldouronate utilization gene cluster from Paenibacillus sp. strain JDR-2. J. Bacteriol., 189, 8863-8870 (2007).

39) G. Nong, J.D. Rice, V. Chow and J.F. Preston: Aldouronate utilization in Paenibacillus sp. strain JDR-2: Physiological and enzymatic evidence for coupling of extracellular depolymerization and intracellular metabolism. Appl. Environ. Microbiol., 75, 4410-4418 (2009).
40) Y. Han, V. Agarwal, D. Dodd, J. Kim, B. Bae, R.I. Mackie, S.T. Nair and I.K.O. Cann: Biochemical and structural insight into xylan utilization by the thermophilic bacterium Caldanaerobius polysaccharolyticus. J. Biol. Chem., 287, 34946-34960 (2012).

41) M. Ejby, F. Fredslund, A. Vijicic-Zagar, B. Svensson, D.J. Slotboom and M.A. Hachem: Structural basis for arabinoxylooligosaccharides capture by the probiotic Bifidobacterium animalis subsp. lactis Bl-04. Mol. Microbiol., 90, 1100-1112 (2013).

42) Z. Krátky and P. Biely: Inducible $\beta$-xyloside permease as a constituent of the xylan-degrading enzyme system of the yeast Cryptococcus albidus. Eur. J. Biochem., 112, 367-373 (1980).

43) H. Tsujibo, M. Kosaka, S. Ikenishi, T. Sato, K. Miyamoto and Y. Inamori: Molecular characterization of a high affinity xylobiose transported of Streptomyces thermoviolaceus OPC-520 and its transcriptional regulation. J. Bacteriol., 186, 1029-1037 (2004).

44) O. Alalouf, Y. Balazs, M. Bvolkinshtein, Y. Grimpel, G. Shoham and Y. Shoham: A new family of carbohydrate esterases is represented by a GDLS hydrolase/acetylxylan esterase from Geobacillus stearothermophilus. J. Biol. Chem., 86, 41993-42001 (2011).

45) I. Krastanova, C. Guarnaccia, S. Zahariev, G. Degrassi and D. Lamba: Heterologous expression, purification, crystallization, X-ray analysis and phasing of the acetyl xylan esterase from Bacillus pumilus. Biochim. Biophys. Acta, 1748, 222-230 (2005).

46) M. Levisson, G.W. Han, M.C. Deller, Q. Xu, P. Biely, S. Hendriks, L.F. Ten Eyck, C. Flensburg, P. Roversi, D.M. Miller, D. McMullan, F. von Delft, A. Kreusch, A.M. Deacon, J. van der Oost, S.A. Lesley, M.A. Elsliger, S.W.M. Kengen and I.A. Wilson: Functional and structural characterization of a thermostable acetyl esterase from Thermotoga maritima. Proteins: Struct. Funct. Bioinform., 80, 1545-1559 (2012).

47) M. Levisson, L. Sun, S. Hendriks, P. Swinkels, T. Akveld, J.B. Bultema, A. Barendregt, R.H.H. van den Heuvel, B.W. Dijkstra, J. van der Oost and S.W.M. Kengen: Crystal structure and biochemical properties of a novel thermostable esterase containing an immunoglobulin-like domain. J. Mol. Biol., 385, 949-962 (2009).

48) P. Biely, M. Mastihubová, M. Tenkanen, J. Eyzaguirre, X.-L. Li and M. Vršanská: Action of xylan deacetylating enzymes on monoacetyl derivatives of 4-nitrophenyl glycosides of $\beta$-D-xylopyranose and $\alpha$-L-arabinofuranose. J. Biotechnol., 151, 137-142 (2011).

49) C. Altaner, B. Saake, M. Tenkanen, J. Eyzaguirre, C.B. Faulds, P. Biely, L. Viikari, M. Siika-aho and J. Puls: Regioselective deacetylation of cellulose acetates by acetyl xylan esterases of different CE-families. J. Biotechnol., 105, 95-104 (2003).

50) P. Biely, M. Mastihubová, G.L. Côté and R.V. Greene: Mode of action of acetylxylan esterase from Streptomyces lividans: A study with deoxy and deoxy-fluoro analogues of acetylated methyl $\beta$-D-xylopyranoside. Biochim. Biophys. Acta, 1622, 8288 (2003).

51) M. Mastihubová and P. Biely: Deoxy and deoxyfluoro analogues of acetylated methyl $\beta$-D-xylopyranoside - substrates for acetylxylan esterases. Carbohydr. Res., 339, 2101-2110 (2004).

52) P. Biely, M. Mastihubová and V. Puchart: The vicinal hydroxyl group is prerequisite for metal activation of Clostridium thermocellum acetylxylan esterase. Biochim. Biophys. Acta, 1770, 565570 (2007)

53) L. Pouvreau, M.C. Jonathan, M.A. Kabel, S.W. Hinz, H. Gruppen and H.A. Schols: Characterization and mode of action of two acetyl xylan esterases from Chrysosporium lucknowense C1 active towards acetylated xylans. Enzyme Microb. Technol., 49, 312-320 (2011).

54) I. Uhliariková, M. Vršanská, B.V. McCleary and P. Biely: Positional specifity of acetylxylan esterases on natural polysaccharide: An NMR study. Biochim. Biophys. Acta, 1830, 33653372 (2013).

55) P. Biely, M. Cziszárová, I. Uhliariková, J.W. Agger, X.-L. Li, V.G.H. Eijsink and B. Westereng: Mode of action of acetylxylan esterases on acetyl glucuronoxylan and acetylated oligosaccharides generated by a GH10 endoxylanase. Biochim. Biophys. Acta, 1830, 5075-5086 (2013).

56) E.J. Taylor, J.P. Turkenburg, T.M. Gloster, F. Vincent, A.M. Brzozowski, C. Dupont, F. Shareck, M.S.J. Centeno, J.A.M. Prates, V. Puchart, L.M.A. Ferreira, C.M.G.A. Fontes, P. Biely 
and G. Davies: Structure and activity of two metal-ion dependent acetyl xylan esterases involved in plant cell wall degradation reveals a close similarity to peptidoglycan deacetylases. J. Biol. Chem., 281, 10968-10975 (2006).

57) P. Biely and G.L. Côté: Microbial hemicellulolytic carbohydrate esterases. in Handbook of Industrial Catalysis, C.T. Hou, ed., Taylor \& Francis Group, Boca Raton, pp. 21-1-21-24 (2005).

58) F. Caufrier, A. Martinou, C. Dupont and V. Bouriotis: Carbohydrate esterase family 4 enzymes: substrate specificity. Carbohydr. Res., 338, 687-692 (2003).

59) N. Hakulinen, M. Tenkanen and J. Rouvinen: Three-dimensional structure of the catalytic core of acetylxylan esterase from Trichoderma reesei: Insights into the deacetylation mechanism. $J$. Struct. Biol., 132, 180-190 (2000).

60) O.B. Siguier, M. Haon, V. Nahoum, M. Marcellin, O. BurletSchiltz, P.M. Coutinho, B. Henrissat, L. Mourey, M. J. O'Donohue, J.-G. Berrin, S. Tranier and C. Dumon: First structural insights into $\alpha$-L-arabinofuranosidases from the two GH62 glycoside hydrolase subfamilies. J. Biol. Chem., 289, 5261-5273 (2014).

61) T. Maehara, Z. Fujimoto, H. Ichinose, M. Michikawa, K. Harazono and S. Kaneko: Crystal structure and characterization of the glycoside hydrolase family $62 \alpha$-L-arabinofuranosidase from Streptomyces coelicolor. J. Biol. Chem., 289, 7962-7972 (2014).

62) P. Biely, C.R. MacKenzie, J. Puls and H. Schneider: Cooperativity of esterases and xylanases in the enzymic degradation of acetyl xylan. Bio/Technol., 4, 731-733 (1986).

63) M.J. Selig, E.P. Knoshaug, W.S. Adney, M.E. Himmel and S.R. Decker: Synergistic enhancement of cellobiohydrolase performance on pretreated corn stover by addition of xylanase and esterase activities. Bioresour. Technol., 99, 4997-5005 (2008).

64) J. Zhang, M. Siika-aho, M. Tenkanen and L. Viikari: The role of acetyl xylan esterase in the solubilisation of xylan and enzymatic hydrolysis of wheat straw and giant reed. Biotechnol. Biofuels, $\mathbf{4}$, 60 (2011).

65) S. Tsujiyama and H. Ueno: Performance of wood-rotting fungibased enzymes on enzymatic saccharification of rice straw. J. Sci. Food. Agric., 93, 2841-2848 (2013).

66) S. Koutaniemi, M.P. van Gool, M. Juvonen, J. Jokela, S.W. Hinz, H.A. Schools and M. Tenkanen: Distinct roles of carbohydrate esterase family CE16 acetyl esterases and polymer-acting acetyl xylan esterases in xylan degradation. J. Biotechnol., 168, 684692 (2013).

67) M. Mastihubová and P. Biely: Lipase-catalysed preparation of acetates of 4-nitrophenyl $\beta$-D-xylopyranoside and their use in kinetic study of acetyl migration. Carbohydr. Res., 339, 13531360 (2004).

68) L. Kremnický and P. Biely: Unique mode of acetylation of oligosaccharides in aqueous two-phase system by Trichoderma reesei acetyl esterase. J. Mol. Catal. B: Enzym., 37, 72-78 (2005).

69) K. Poutanen and M. Sundberg: An acetyl esterase of Trichoderma reesei and its role in the hydrolysis of acetyl xylans. Appl. Microbiol. Biotechnol., 28, 419-424 (1988).

70) X.-L. Li, C.D. Skory, M.A. Cotta, V. Puchart and P. Biely: Novel family of carbohydrate esterases, bases on identification of the Hypocrea jecorina acetyl esterase gene. Appl. Environ. Microbiol., 74, 7482-7489 (2008).

71) P. Biely, M Cziszárová, J.W. Agger, X.-L. Li, V. Puchart, M. Vršanská, V.G.H. Eijsink and B. Westereng: Trichoderma reesei CE16 acetyl esterase and its role in enzymatic degradation of acetylated hemicelluloses. Biochim. Biophys. Acta, 1840, 516525 (2014) 